\title{
Smartphone Applications for Iranian Users with Hypertension
}

\author{
A Safari ${ }^{1,}{ }^{*}$; A Sheikhtaheri ${ }^{2}$; Z Hasani ${ }^{3}$ \\ ${ }^{1}$ MSc. in Health Information Technology, Researcher, School of Allied Medical Sciences, Kermanshah University of Medical Sciences, Kermanshah, Iran \\ ${ }^{2}$ Assistant Professor, Department of Health Information Management, School of Health Management and Information Sciences, Iran University of Medical Sciences, Tehran, Iran \\ ${ }^{3}$ MSc. in Medical Informatics, School of Allied Medical Sciences, Tehran University of Medical Sciences, Tehran, Iran \\ ${ }^{*}$ Corresponding author: A safari, MSc. in Health Information Technology, Researcher, School of Allied Medical Sciences, Kermanshah University of Medical Sciences, Kermanshah, \\ Iran. Tel:+98-9381330118, E-mail: safari.ameneho@gmail.com
}

Received: 11 Dec 2016

Accepted: 01 Jan 2017

Epub: 23 Feb 2017

Ppub: 15 Jan 2018

\begin{abstract}
Background: The development of medical and health applications has increased dramatically over the world due to the widespread penetration of mobile network. It seems that mobile health technology has become a leading technology in the world. Meanwhile, the bulk of applications have been designed for managing hypertension.

Objectives: This study aims to present a picture of the current state of the smartphone application market for Iranian patients with were done.

Methods: Between September until October of 2016, medical categories in cafe bazar's website (for Android OS) as well as Sibche website (for the operating system iOS), Google Play (for Android OS), and IranApps that have reviews and applications designed for patients with hypertension were identified in Farsi. Finally, the attributes associated with these applications with Microsoft Excel 2010 were evaluated.

Results: The results showed that 25 apps from the café bazar were assigned to hypertension, on IranApps there was an app dedicated to high blood pressure, and no apps regarding blood pressure was found on the Sibche website as well as Google Play. Among these applications, 20 (92.76\%) delivered content to explore ways in preventing and controlling the disease as well as hypertension. Only 6 (07.23\%) were about daily record blood pressure and submit it in the form of reports and graphs. Among these 26 applications, 4 apps (30.15\%) were for sale and 22 apps (61.84\%) were free.

Conclusions: A limited number of Iranian applications, in the field of hypertension and lack of completeness in order to meet the needs of patients, represent that unlike global development for these apps in Iran, the needs of patients with hypertension are not considered. In addition, most of the apps have duplicate content. Therefore the absence of doctors and specializing in the design and development of applications raises concerns about user safety.

Keywords: mHealth; Hypertension; Application
\end{abstract}

